\title{
Surfactant protein A genetic variants associate with severe respiratory insufficiency in pandemic influenza A virus infection
}

Estefanía Herrera-Ramos 1,2, Marta López-Rodríguez', José Juan Ruíz-Hernández³, Juan Pablo Horcajada 4,5, Luis Borderías ${ }^{6}$, Elisabeth Lerma ${ }^{4}$, José Blanquer ${ }^{7}$, María Carmen Pérez-González ${ }^{8}$, María Isabel García-Laorden ${ }^{1,9}$, Yanira Florido ${ }^{1,2}$, Virginia Mas-Bosch ${ }^{10}$, Milagro Montero ${ }^{4}$, José María Ferrer ${ }^{11}$, Luisa Sorlí ${ }^{4}$, Carlos Vilaplana ${ }^{10}$, Olga Rajas ${ }^{12}$, Marisa Briones ${ }^{13}$, Javier Aspa ${ }^{12}$, Eduardo López-Granados ${ }^{14}$, Jordi Solé-Violán ${ }^{11}$, Felipe Rodríguez de Castro ${ }^{2,15}$ and Carlos Rodríguez-Gallego ${ }^{1,2^{*}}$

\begin{abstract}
Introduction: Inherited variability in host immune responses influences susceptibility and outcome of Influenza A virus (IAV) infection, but these factors remain largely unknown. Components of the innate immune response may be crucial in the first days of the infection. The collectins surfactant protein (SP)-A1, -A2, and -D and mannose-binding lectin (MBL) neutralize IAV infectivity, although only SP-A2 can establish an efficient neutralization of poorly glycosylated pandemic IAV strains.
\end{abstract}

Methods: We studied the role of polymorphic variants at the genes of MBL (MBL2), SP-A1 (SFTPA1), SP-A2 (SFTPA2), and SP-D (SFTPD) in 93 patients with H1N1 pandemic 2009 (H1N1pdm) infection.

Results: Multivariate analysis showed that two frequent SFTPA2 missense alleles (rs1965708-C and rs1059046-A) and the SFTPA2 haplotype $1 A^{\circ}$ were associated with a need for mechanical ventilation, acute respiratory failure, and acute respiratory distress syndrome. The SFTPA2 haplotype $1 A^{7}$ was a protective variant. Kaplan-Meier analysis and Cox regression also showed that diplotypes not containing the $1 A^{\prime}$ haplotype were associated with a significantly shorter time to ICU admission in hospitalized patients. In addition, rs1965708- $C(P=0.0007)$, rs 1059046- $A(P=0.0007)$, and haplotype $1 A^{0}(P=0.0004)$ were associated, in a dose-dependent fashion, with lower $\mathrm{PaO}_{2} / \mathrm{FiO}_{2}$ ratio, whereas haplotype $1 A^{1}$ was associated with a higher $\mathrm{PaO}_{2} / \mathrm{FiO}_{2}$ ratio $(P=0.001)$.

Conclusions: Our data suggest an effect of genetic variants of SFTPA2 on the severity of H1N1pdm infection and could pave the way for a potential treatment with haplotype-specific $\left(1 A^{1}\right)$ SP-A2 for future IAV pandemics.

\section{Introduction}

Influenza A virus (IAV) infection is usually a mild and self-limited condition. Likewise, infection with the H1N1 pandemic 2009 (H1N1pdm) IAV often results in an uncomplicated flu, but, in a small subset of patients, it may rapidly evolve to primary viral pneumonia, acute respiratory failure (ARF), and acute respiratory distress

\footnotetext{
* Correspondence: jrodgal@gobiernodecanarias.org

'Department of Immunology, Hospital Universitario de Gran Canaria Dr. Negrín, Las Palmas de Gran Canaria 35010, Spain

2Department of Medical and Surgical Sciences, School of Medicine, Universidad de Las Palmas de Gran Canaria, Las Palmas de Gran Canaria 35016, Spain

Full list of author information is available at the end of the article
}

syndrome (ARDS) [1]. Inherited and acquired variability in host immune responses may influence susceptibility and outcome of IAV infection, although these factors remain largely unknown [2-4].

Before exposure to H1N1pdm IAV, most individuals, particularly those born after 1957, lack serum antibodies capable of neutralizing the virus [1]. Adaptive immune responses, which are needed for ultimate viral clearance, take several days to develop. Therefore, components of the innate immunity that are able to neutralize IAV infection with minor inflammation may be crucial for host defense in the first few days after infection. Different soluble pattern-recognition molecules of the innate immunity 
can neutralize IAV infection. Several secreted human Ctype lectins of the collectin family, the serum mannosebinding lectin (MBL), the pulmonary surfactant proteins (SP) $-\mathrm{A} 1,-\mathrm{A} 2$, and $-\mathrm{D}$, and collectin 11 (CL-11, alias collectin kidney $1, \mathrm{CL}-\mathrm{K} 1$ ) may neutralize IAV infectivity in vitro [5-8]. Among these collectins, the SPs have been shown to exert an important role against IAV infection in animal models: mice lacking SP-A or SP-D have increased susceptibility to IAV infection, and their role seem to depend on IAV strains, particularly pandemic versus seasonal strains [9-14]. In addition, variability at the collectin genes, MBL2 (Ensembl: ENSG00000165471), SFTPA1 (Ensembl: ENSG00000122852), SFTPA2 (Ensembl: ENSG00000185 303), and SFTPD (Ensembl: ENSG00000133661) have been found to be associated with susceptibility to and/or severity of several bacterial and viral infectious diseases [6,15].

It hence follows that these collectins are firm candidates to explain, at least in part, the role of host genetic variability in the defense against IAV infection. The human SP-A locus consists of two similar genes, SFTPA1 and SFTPA2, localized within a cluster (10q21-24) that includes the SP-D gene (SFTPD) [16]. MBL2 was reported not to be in physical linkage with the genes of these SPs [17], but linkage disequilibrium of $M B L 2$ with SFTPA1 and SFTPA2 has been shown; and LD among variants at these genes could influence the results of geneticassociation studies $[6,18,19]$.

In the present study, we assessed the role of variants at the SFTPA1, SFTPA2, and SFTPD genes in H1N1pdm IAV infection in humans. Variants at the neighbor collectin gene $M B L 2$ were also analyzed.

\section{Materials and methods H1N1pdm-infected patients}

We recruited 124 patients with H1N1pdm infection between July 2009 and November 2011. Thirty-one individuals with ancestors other than Spanish were excluded. Of 93 unrelated white Spanish patients, 70 were hospitalized at five tertiary Spanish hospitals, and 23 were attended at primary care centers (Figure 1). Data and samples from all ambulatory patients and from $30 \%$ of hospitalized individuals were retrospectively obtained; in the remaining patients, data were obtained prospectively. All patients were treated with oseltamivir, and only one patient had been previously vaccinated against H1N1pdm.

Diabetes, previous lung disease, solid-organ transplantation, immunosuppression, body mass index (BMI) $\geq 30$, human immunodeficiency virus (HIV) infection, and pregnancy were considered risk factors. Sepsis, septic shock, and multiorgan dysfunction syndrome (MODS) were defined by using the American College of Chest Physicians/ Society of Critical Care Medicine criteria [20]. ARF and ARDS were diagnosed according to the American European Consensus Conference Definition [21]. Functional parameters of gas exchange were calculated on the basis of the ratio of oxygen arterial pressure to oxygen inspiratory fraction $\left(\mathrm{PaO}_{2} / \mathrm{FiO}_{2}\right)$. In hospitalized patients without arterial blood gas analysis $(n=18)$, ARF was established when hemoglobin oxygen saturation, breathing room air, was lower than $90 \%$. In patients with intensive care unit (ICU) admission, severity of illness was evaluated with the Acute Physiology and Chronic Health Evaluation II score, taking the worst reading of the first 24 hours in the ICU.

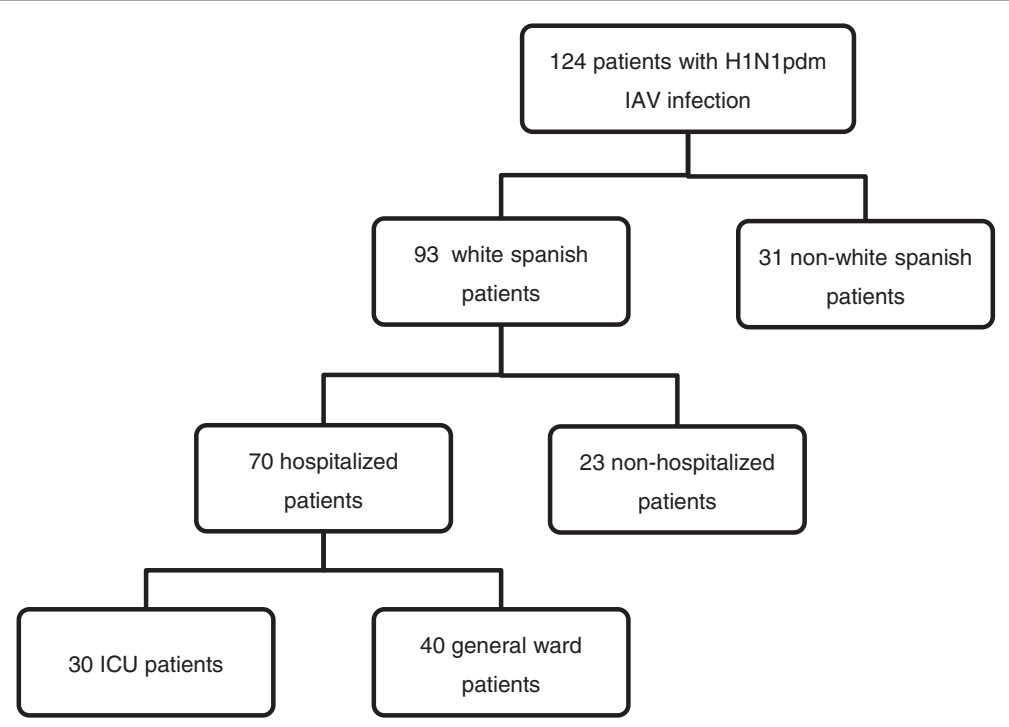

Figure 1 Selection process for patients with H1N1pdm 2009 infection. Infection by H1N1pdm was confirmed in all the 124 patients. 
All steps were performed in complete accordance to the Helsinki declaration. The protocol was approved by Clinical Research Ethics Committees (CEIC) of hospitals involved (CEIC Hospital Universitario de Gran Canaria Doctor Negrín, Las Palmas de Gran Canaria; CEIC Hospital San Jorge, Huesca and CEIC Hospital Clínico y Universitario de Valencia, Valencia). Informed consent was obtained of all patients included.

\section{Detection of $\mathrm{H} 1 \mathrm{~N} 1 \mathrm{pdm}$ by real-time polymerase chain reaction}

Influenza A H1N1 virus in the 124 patients was detected in nasopharyngeal swabs by using the Real-Time ready Influenza A (H1N1) Detection Set (Roche Diagnostics $\mathrm{GmbH}$, Mannheim, Germany) according to manufacturer's protocol.

\section{General Spanish-population subjects}

The general population group consisted of white unrelated Spanish healthy volunteers (blood and bone marrow donors as well as hospital staff) from four tertiary Spanish hospitals. The control group was analyzed for MBL2 $(n=1,736)$ and SFTPA1 and SFTPA2 $(n=769)$ polymorphisms in previous studies $[18,19]$. For the nine SFTPD variants under study, 963 individuals from the general population group recruited at the same hospitals were analyzed in this study. The protocol was approved by Clinical Research Ethics Committees (CEIC) of hospitals involved (CEIC Hospital Universitario de Gran Canaria Doctor Negrín, Las Palmas de Gran Canaria; CEIC Hospital de la Princesa, Madrid; CEIC Hospital San Jorge, Huesca and CEIC Hospital Clínico y Universitario de Valencia, Valencia). Informed consent was obtained of the general-population individuals included. No data about whether individuals from the general Spanish population group were or were not infected with H1N1pdm were available. Foreigners and individuals with ancestors other than Spanish were previously excluded.

\section{Selection of single-nucleotide polymorphisms}

Deficient and low MBL serum levels are mainly due to the presence of three common point mutations in the exon 1 of the $M B L 2$ gene: alleles $B$ (rs1800450), $C$ (rs1800451), and $D$ (rs5030737) are termed $O$ alleles, $A$ being the wildtype. Serum MBL levels are very low or absent in individuals homozygous for $O$ alleles. In addition, the presence of the promoter allele $X$ (rs7096206) has an important downregulating effect, and individuals with $X A / O$ also have very low MBL serum levels. $O / O$ together with $X A / O$ genotypes are considered MBL-deficient genotypes, which are common in most populations.

The human SP-A locus consists of two similar genes, SFTPA1 and SFTPA2, located on chromosome 10q22.3, within a cluster that includes the SP-D gene (SFTPD). In addition, a certain degree of linkage disequilibrium (LD) exists among SP genes and the MBL gene (10q21-24) $[18,19]$. On the basis of the existence of several SNPs, SP-A haplotypes are conventionally denoted as $6 A^{n}$ for the SFTPA1 gene (V19A, rs1059047; L50V, rs1136450; R219W, rs4253527) and $1 A^{n}$ for the SFTPA2 gene (T9N, rs1059046; A91P, rs17886395; Q223K, rs1965708) [22]. These missense single-nucleotide polymorphisms (SNPs) at SFTPA1 and SFTPA2 were analyzed in our study. The most frequent conventional haplotypes of these genes, except $1 A$ and $1 A^{5}$, can be unambiguously identified.

For the analysis of SFTPD, genotypic data of individuals of European ancestry (CEU) from the International HapMap Project [23] were used to select LD tagging SNPs. Pairwise LD-tagging was achieved with Haploview v. 4.2 [24] for SNPs with a minimum minor allele frequency of 0.05 and $r^{2}$ of 0.8 . As result, three intronic SNPs (rs10887199, rs7078012, and rs723192) and one synonymous SNP (rs6413520) were selected. Three missense SNPs were also analyzed: rs3088308 (S290T), rs2243639 (T180A), and rs721917 (M31T). Additionally, we studied an SNP in the SFTPD promoter region (rs1885551) that was recently reported to have a profound impact on serum SP-D levels [25] and an intronic SNP (rs17886286) associated with susceptibility to invasive pneumococcal disease [26].

\section{DNA extraction and genotyping}

Genomic DNA was extracted from $400 \mu \mathrm{l}$ of peripheral blood by using iPrep PureLink gDNA Blood kit in the iPrep Purification Instrument (Invitrogen by Life Technologies, Carlsbad, CA, USA). Extracted DNA integrity was checked by NanoDrop ND-1000 (NanoDrop Technologies, Wilmington, DE, USA). In total, 19 polymorphisms at MBL2, SFTPA1, SFTPA2 and SFTPD were studied.

MBL2, SFTPA1, SFTPA2, and SFTPD rs721917 SNPs were analyzed by means of PCR-RFLP and PCR-SSP techniques, as previously described $[18,19]$. The other eight SNPs of SFTPD were determined by predesigned Taqman SNP genotyping assays (Assays IDs: C_ 26726209_10, C_26726205_10, C_31530298_10, C_63652102_10, C_29213175_10, C__1362981_20, C__630297_10, and C_12124527_20), according to the manufacturer's protocol, with commercially available reagents by means of ViiA $^{\mathrm{rm}} 7$ Real-time PCR System (Applied Biosystems, Foster City, CA, USA).

\section{Data analysis}

The Hardy-Weinberg equilibrium was analyzed with Haploview v. 4.2. Haplotypes were estimated in silico with PHASE v. 2.1.1 under 1,000 permutations. Statistical analyses were performed by using SPSS 20.0 (SPSS, Inc., Chicago, IL, USA). The comparison of all genotype 
distributions based on susceptibility and severity was performed by using the $x^{2}$ test or Fisher Exact test when needed, and odds ratios with 95\% confidence intervals were calculated. The relation between severity in hospitalized patients and genotypes was evaluated by binary logistic regression models: age, gender, risk factors, and secondary infection (either bacteremia or secondary bacterial pneumonia) were included as independent variables.

Comparison of the $\mathrm{PaO}_{2} / \mathrm{FiO}_{2}$ ratio according to genetic variants was performed by a using linear regression model adjusted by age, gender, risk factors, and secondary infection (either bacteremia or secondary bacterial pneumonia).

To assess the need for ICU admission, a multivariate analysis was performed, including the aforementioned variables, by conditional logistic regression stratified by hospital of origin.

Log-rank (LR) $X^{2}$ tests were calculated to compare ICU admission according to the distribution of genetic variants. Cox proportional hazard ratios adjusted for the independent variables age, gender, risk factors, development of secondary bacterial infection (pneumonia or bacteremia), and hospital of origin were also performed.

\section{Results}

Demographic and clinical characteristics of patients are shown in Table 1. The genotype distribution of SNPs did not differ significantly under conditions of HardyWeinberg equilibrium in all of the groups studied (data not shown). Frequencies of the genetic variants under analysis were not found to be significantly different between H1N1pdm-infected patients and the general population (Table 2).

When severity of infection was evaluated in hospitalized patients $(\mathrm{n}=70)$ (Table 3), we observed that two missense variants at SFTPA2 (rs1965708-C and rs1059046-A) were significantly associated with the need for mechanical ventilation (MV) and with development of ARF and ARDS. Most of these associations remained significant after multivariate analysis adjusted for age, gender, risk factors, and secondary infection (either bacteremia or secondary bacterial pneumonia). The alleles rs1965708- $C$ and rs1059046- $A$ were overrepresented in patients requiring $\mathrm{MV}(P=0.003$; OR, 2.43; and $P=0.016$; OR, 3.71, respectively), and in those who developed ARF $(P=0.006 ;$ OR, 4.09; and $P=0.005$; OR, 3.70 , respectively) or ARDS $(P=0.006$; OR, 17.68; and $P=0.016$; OR, 3.71, respectively). The variant rs1965708- $C$ was also associated with septic shock $(P=0.007$; OR, 17.16). The SFTPA1 allele rs1136450-G was also associated, although to a lower extent, with the development of ARF $(P=0.038$; OR, 2.64) and the use of MV $(P=0.040$; OR, 2.55); these associations may be secondary to the existence of LD between SFTPA1 and SFTPA2 variants (Figure 2).
Table 1 Demographic and clinical characteristics of H1N1 pdm-infected patients

\begin{tabular}{lcc}
\hline Characteristics & \multicolumn{3}{c}{ Subjects } \\
\hline Age (years) & \multicolumn{3}{c}{$43.5 \pm 18.0^{\mathrm{a}}$} \\
Gender (male) & 52 & $(55.9)$ \\
Hospital admission & & \\
$\quad$ No & 23 & $(24.7)$ \\
$\quad$ Yes & 70 & $(75.3)$ \\
PVP & & \\
$\quad$ No & 40 & $(43.0)$ \\
Yes & 53 &
\end{tabular}

ICU admission

$\mathrm{No}$

ARF

Shock

ARDS

MODS

MV

Hospital mortality

Risk factor

Secondary bacterial infection ${ }^{\mathrm{b}}$

ARDS, acute respiratory distress syndrome; ARF, acute respiratory failure; ICU, intensive care unit; MODS, multiorgan dysfunction syndrome; MV, mechanical ventilation; PVP, Primary viral pneumonia.

${ }^{a}$ Data are presented as mean \pm SD or number of individuals (\%). ${ }^{b}$ Ten patients had secondary bacterial pneumonia, and seven patients had bacteremia (four of them with secondary bacterial pneumonia).

When haplotypes, based on combinations of missense SNP, were analyzed (Table 4), the SFTPA2 haplotype $1 A^{\circ}$ was found to be associated with the need for MV and with the development of ARF and ARDS in hospitalized patients. These differences were found to be independent of age, 
Table 2 Distribution of genotype frequencies of collectin genes in general Spanish population and H1N1pdm-infected patients

\begin{tabular}{|c|c|c|c|}
\hline Variants & Genotypes & General Spanish population & H1N1pdm-infected patients \\
\hline$M B L 2^{\mathrm{a}}$ & & $n=1736$ & $n=93$ \\
\hline \multicolumn{4}{|l|}{ rs1800451 (G57E) } \\
\hline rs1800450 (G54D) & $\mathrm{AA} / \mathrm{AO} / \mathrm{OO}$ & $1,032(59.4) / 615(35.4) / 89(5.1)$ & $54(58.1) / 36(38.7) / 3(3.2)$ \\
\hline \multicolumn{4}{|l|}{ rs5030737 (R52C) } \\
\hline \multicolumn{4}{|l|}{ rs7096206 (Prom) } \\
\hline MBL deficiency & $A A+Y A O / X A O+O O$ & $1,475(85.0) / 261(15,0)$ & $77(82.8) / 16(17.2)$ \\
\hline SFTPA2 & & $n=769$ & $n=93$ \\
\hline rs1965708 (Q223K) & $\mathrm{AA} / \mathrm{CA} / \mathrm{CC}$ & $22(2.9) / 244(31.7) / 503(65.4)$ & $4(4.3) / 33(35.5) / 56(60.2)$ \\
\hline rs17886395 (A91P) & GG/GC/CC & $623(81.0) / 134(17.4) / 12(1.6)$ & $72(77.4) / 19(20.4) / 2(2.2)$ \\
\hline rs1059046 (T9N) & $C C / A C / A A$ & $97(12 ., 6) / 349(45.4) / 323(42.0)$ & $14(15.1) / 48(51.6) / 31$ (33.3) \\
\hline SFTPA1 & & $n=769$ & $n=93$ \\
\hline rs1059047 (V19A) & TT/TC/CC & $680(88.4) / 88(11.4) / 1(0.1)$ & $82(88.2) / 10(10.8) / 1(1.1)$ \\
\hline rs1136450 (L50V) & $\mathrm{CC} / \mathrm{GC} / \mathrm{GG}$ & $117(15.2) / 334(43.4) / 318(41.4)$ & $12(12.9) / 48(51.6) / 33(35.5)$ \\
\hline rs4253527 (R219W) & $\mathrm{CC} / \mathrm{CT} / \mathrm{TT}$ & $620(80.6) / 142(18.5) / 7(0.9)$ & $76(81.7) / 16(17.2) / 1(1.1)$ \\
\hline SFTPD & & $n=963$ & $n=93$ \\
\hline rs3088308 (S290T) & AA/AT/TT & $829(86.1) / 129(13.4) / 5(0.5)$ & $77(82.8) / 14(15.1) / 2(2.1)$ \\
\hline rs2243639 (T180A) & ТT/TC/CC & $373(38.7) / 449(46.6) / 141$ (14.6) & $42(45.2) / 42(45.2) / 9(9.6)$ \\
\hline rs10887199 (Intr) & TT/TC/CC & 759 (78.8)/189 (19.6)/15 (1.6) & $71(76.3) / 20(21.5) / 2(2.2)$ \\
\hline rs17886286 (Intr) & $\mathrm{CC} / \mathrm{CG} / \mathrm{G}$ & $828(86.0) / 126(13.1) / 9(0.9)$ & $79(85.0) / 13(14.0) / 1(1.0)$ \\
\hline rs7078012 (Intr) & $\mathrm{CC} / \mathrm{CT} / \mathrm{TT}$ & $629(65.3) / 292(30.3) / 42(4.4)$ & $52(55,.) / 38(40.9) / 3(3.2)$ \\
\hline rs6413520 (S45S) & $\mathrm{AA} / \mathrm{AG} / \mathrm{GG}$ & $824(85.6) 134(13.9) / 5(0.5)$ & $82(88.2) / 11(11.8) / 0(0.0)$ \\
\hline rs721917 (M31T) & ТТ/TC/CC & $356(37.0) / 438(45.5) / 169(17.5)$ & $36(38 / .7) / 38(40.9) / 19(20.4)$ \\
\hline rs723192 (Intr) & $\mathrm{CC} / \mathrm{CT} / \mathrm{TT}$ & 757 (78.6)/195 (20.3)/11 (1.1) & $70(75.3) / 21(22.6) / 2(2.1)$ \\
\hline rs1885551 (Prom) & $\mathrm{AA} / \mathrm{AG} / \mathrm{GG}$ & $766(79.5) / 182(18.9) / 15(1.6)$ & $72(77.4) / 19(20.4) / 2(2.2)$ \\
\hline
\end{tabular}

${ }^{\mathrm{a}} \mathrm{O} / \mathrm{O}$ together with $\mathrm{XA} / \mathrm{O}$ genotypes are considered MBL-deficient genotypes. Intr, intronic region; Prom, promoter region. SNPs were added on the basis of chromosome position.

gender, risk factors, and secondary infection (either bacteremia or secondary bacterial pneumonia) in multivariate analysis: $P=0.003$ (data in Table 4), OR 3.73; $P=0.022$; OR, 2.81; and $P=0.016$; OR, 3.41, for the need for MV, ARF, and ARDS comparisons, respectively. By contrast, the SFTPA2 haplotype $1 A^{1}$ was found to be protective in a dominant model against the requirement for $\mathrm{MV}$ and the development of $\mathrm{ARF}$ and ARDS in the multivariate analysis $(P=0.015$; OR, 0.16 ; $P=0.005$; OR, 0.21; and $P=0.021$; OR, 0.12).

As an additional measurement of severity, we analyzed the need for ICU admission in hospitalized patients. The variants rs1965708- $C$ and $\operatorname{rs1059046-~} A$ were associated with a higher rate of ICU admission, although only rs1965708- $C$ remained significant after conditional logistic regression adjustment for the previously mentioned variables and stratified by hospital of origin $(P=0.031$; OR, 2.43) (Table 3). The same analysis remained significant at SFTPA2 haplotype $1 A^{1}$ in a dominant model $(P=0.029$;
OR, 0.32) (Table 4). It is noteworthy that Kaplan-Meier analysis and the log-rank test showed that the variants rs1965708- $C$ and rs1059046- $A$ were associated with a significantly shorter time to ICU admission in hospitalized patients (Figure 3). The same analysis also showed that ICU admission was less frequently required among patients with the haplotype $1 A^{1}$, and this difference was readily detected in the first days after hospitalization. The effect of these variants at the time of ICU admission remained significant in Cox regression.

We finally evaluated the influence of genetic variants of SFTPA1 and SFTPA2 on respiratory gas exchange in 52 hospitalized patients. SFTPA2 alleles (rs1965708-C and $\operatorname{rs} 1059046-A)$ and haplotypes $\left(1 A^{O}\right)$ predisposing to increased severity were found to be associated with significantly lower $\mathrm{PaO}_{2} / \mathrm{FiO}_{2}$ ratios, independent of age, gender, risk factors, secondary bacterial pneumonia, and bacteremia (Figure 4). Interestingly, the effect of these alleles or haplotypes was found to be dependent on the 
Table 3 Severity of H1N1pdm infection in hospitalized patients regarding missense single-nucleotide polymorphisms of SFTPA2 and SFTPA1

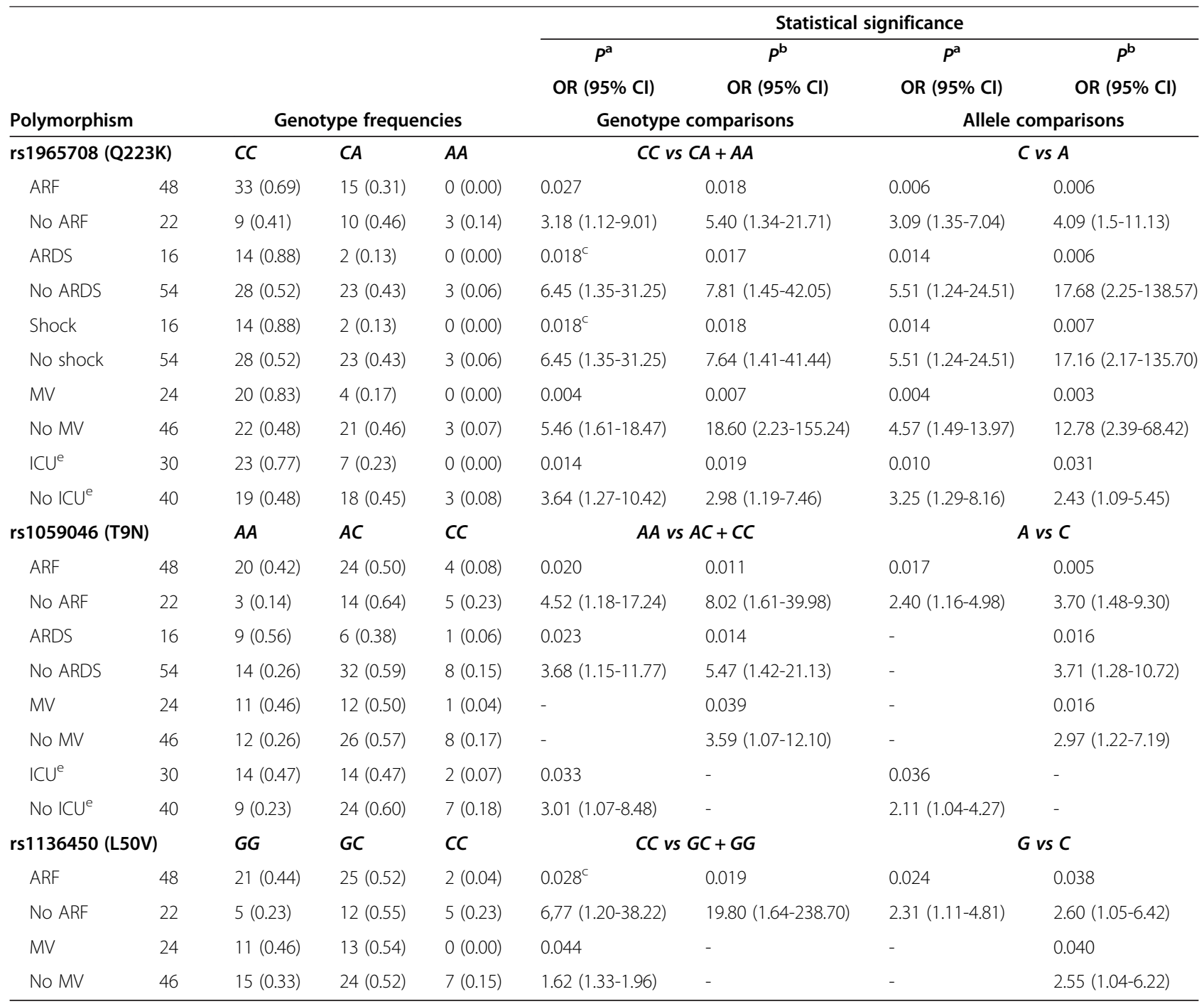

${ }^{a} P$ value for the bivariate comparison calculated with the $x^{2}$ test. ${ }^{b} P$ value for the multivariate analysis calculated with binary logistic regression, including the variables age, gender, risk factors, secondary bacterial pneumonia, and bacteremia. cap value by Fisher Exact test. ${ }^{d_{i p}}$ value for the multivariate analysis calculated with binary logistic regression, including the variables age, gender, risk factors, and secondary bacterial pneumonia (bacteremia variable was excluded because it

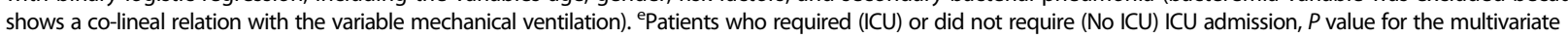
analysis calculated with conditional logistic regression stratified by hospital of origin, including the co variables age, gender, risk factors, secondary bacterial pneumonia, and bacteremia. Only those comparisons with $P<0.05$ and significant odds ratios were included. OR ( $95 \%$ Cl), Odds ratio (95\% confidence interval); ARF, acute respiratory failure; ARDS, acute respiratory distress syndrome; MV, mechanical ventilation; ICU, intensive care unit.

number of alleles present in a genotype or diplotype: $P=0.0007, P=0.0007$ and $P=0.0004$ for $\mathrm{rs} 1965708-C$, rs1059046- $A$, and $1 A^{0}$ respectively. The $1 A^{1}$ haplotype, which was associated with lesser severity, was also associated with higher $\mathrm{PaO}_{2} / \mathrm{FiO}_{2}$ ratios $(P=0.0007)$, and this effect was also found to be dose-dependent in a linear regression model adjusted for the same independent variables $(P=0.0014)$ (Figure 4$)$.

\section{Discussion}

Glycosylation of hemagglutinins may be an important mechanism by which IAV can evade recognition by antibodies in an immune population. By contrast, glycosylation of hemagglutinins is important in determining sensitivity of IAV to recognition by collectins [7,27]. SP-D and MBL bind to mannose-rich glycans on the hemagglutinins and neuraminidase glycoproteins of IAV, agglutinating viral particles and inhibiting infectivity and neuraminidase activity [7]. Among the known human collectins, SP-D has the strongest in vitro capability of aggregating and neutralizing activity of IAV [7,12,27]. Hemagglutinins from all available strains of pandemic influenza viruses show significantly lower glycosylation sites compared with seasonal strains; and pandemic viruses, particularly H1N1pdm, were found 


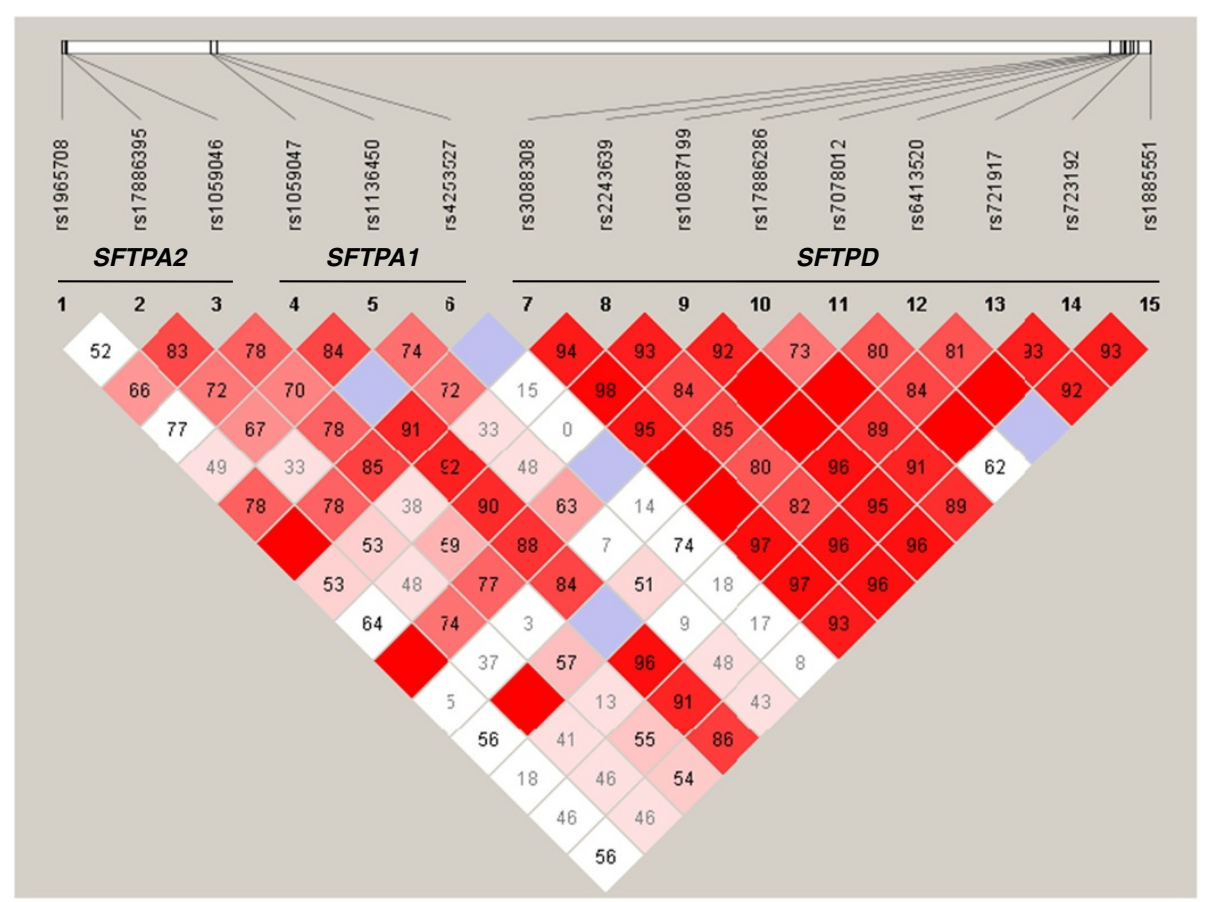

Figure 2 Linkage disequilibrium $\left(D^{\prime}\right)$ among genetic polymorphisms of surfactant proteins in general Spanish population $(N=687)$. $L D$ plots for pair wise $D^{\prime}$ between markers and $D^{\prime}$ values are indicated in percentages within squares in the LD plot. Strong LD is indicated by dark gray/red, whereas light gray/pink and white indicate uninformative and low confidence values, respectively.

to be resistant to the antiviral activities of SP-D, MBL, and the pentraxin PTX3 [7,13,27]. We have not observed any association with severity when deficient-, low-, or high-MBL producer genotypes and SFTPD SNPs or haplotypes were compared (data not shown). Interestingly, SP-A binding to hemagglutinins and SP-A-dependent IAV neutralization in vitro are not influenced by the extent of hemagglutinins glycosylation. SP-A is slightly more effective than SP-D at neutralizing nonglycosylated IAV strains, and it neutralizes IAV strains resistant to SP-D [12].

Accordingly, our results point toward a role of SFTPA2 SNPs and haplotypes, particularly haplotypes $1 A^{O}$ and $1 A^{1}$, in the severity of H1N1pdm infection. The rationale for such an association seems to be the involvement of SFTPA2 variants in gas-exchange parameters, which, in the case of pulmonary IAV infection, is largely dependent on IAV-induced lung inflammation.

Besides its role in IAV neutralization, the hydrophilic SP-A and -D have been shown to have an antiinflammatory function. Binding of the carbohydrate-binding recognition domains (CRDs) to signal-inhibitory regulatory protein $\alpha(\mathrm{SIRP} \alpha)$ on alveolar macrophages suppresses $\mathrm{NF}-\mathrm{KB}$ activation and inflammation, allowing the healthy lung to remain in a quiescent state. SP-A and SP-D can also inhibit inflammation, through the CRD, blocking Toll-like receptors 2 and 4. Pulmonary clearance of IAV was reduced, and pulmonary inflammation and severity were increased in Sftpa-/-mice compared with wildtype mice $[10,11]$. SP-A was also found to disturb the host inflammatory response to IAV infection in mouse models without directly influencing viral growth and spread, and even without demonstrable viral binding, at least when the virus was resistant to neutralization by SP-D [9]. Insufficient amounts of surfactant, particularly SP-A, have been observed in prematurely born infants with respiratory distress syndrome (pRDS). The haplotype $1 A^{O}$ or SFTPA1-SFTPA2 haplotypes containing $1 A^{O}$ have been repeatedly associated with the development of pRDS, whereas SP haplotypes containing $1 A^{1}$ were found to be protective against that condition $[5,15]$. These findings parallel those observed in our study, suggesting that $1 A^{0}$ and $1 A^{1}$ might influence the inflammatory response and the severity of H1N1pdm infection without binding to IAV.

Human SP-A1 and -A2 have similar in vitro hemagglutination-inhibition activity of IAV strains exhibiting non- or poorly glycosylated hemagglutinins heads [14]. However, in all functional assays, SP-A2 is more active than SP-A1 $[5,15]$. So it is not surprising that among all the genetic variants of collectins analyzed in our study, only a few alleles and haplotypes of SFTPA2 were associated with H1N1pdm severity in hospitalized patients. Furthermore, 
Table 4 Severity of H1N1pdm infection in hospitalized patients regarding haplotypes of SFTPA2

\begin{tabular}{|c|c|c|c|c|c|c|c|c|}
\hline \multirow{4}{*}{\multicolumn{2}{|c|}{ Haplotype }} & & & & \multicolumn{4}{|c|}{ Statistical significance } \\
\hline & & & & & $P^{\mathrm{a}}$ & $P^{\mathrm{b}}$ & $P^{a}$ & $P^{\mathbf{b}}$ \\
\hline & & & & & OR $(95 \% \mathrm{Cl})$ & OR $(95 \% \mathrm{Cl})$ & OR $(95 \% \mathrm{Cl})$ & OR $(95 \% \mathrm{Cl})$ \\
\hline & & \multicolumn{3}{|c|}{ Diplotype frequencies } & \multicolumn{2}{|c|}{ Diplotype comparisons } & \multicolumn{2}{|c|}{ Haplotype comparisons } \\
\hline \multicolumn{2}{|l|}{$1 A^{0}(A G C)$} & $1 A^{0} / 1 A^{0}$ & $1 A^{0} /$ rest & Rest/rest & \multicolumn{2}{|c|}{$1 A^{0} / 1 A^{0}$ vs $1 A^{O} /$ rest + rest/rest } & \multicolumn{2}{|c|}{$1 A^{O}$ vs rest } \\
\hline ARF & 48 & $16(0.33)$ & $27(0.56)$ & $5(0.10)$ & 0.031 & 0.045 & 0.023 & 0.022 \\
\hline No ARF & 22 & $2(0.09)$ & $14(0.64)$ & $6(0.27)$ & $5.00(1.04-24.10)$ & $6.38(1.04-38.97)$ & $2.30(1.11-4.76)$ & $2.81(1.16-6.77)$ \\
\hline ARDS & 16 & $8(0.50)$ & $7(0.44)$ & $1(0.06)$ & $0.020^{c}$ & 0.013 & 0.029 & 0.016 \\
\hline No ARDS & 54 & $10(0.19)$ & $34(0.63)$ & $10(0.19)$ & $4,40 .(1.33-14.56)$ & $5.44(1.43-20.77)$ & $2.56(1.08-6.02)$ & $3.41(1.26-9.22)$ \\
\hline MV & 24 & $11(0.46)$ & $12(0.50)$ & $1(0.04)$ & 0.005 & 0.004 & 0.007 & 0.003 \\
\hline No MV & 46 & $7(0.15)$ & $29(0.63)$ & $10(0.22)$ & $4.72(1.51-14.69)$ & $7.03(1.83-26.95)$ & $2.78(1.31-5.83)$ & $3.73(1.55-9.00)$ \\
\hline \multicolumn{2}{|l|}{$1 A^{1}(C G A)$} & $1 A / 1 A^{1}$ & $1 A^{1} /$ rest & rest/rest & \multicolumn{2}{|c|}{$1 A^{1} / 1 A^{1}+1 A^{1} /$ rest vs rest/rest } & \multicolumn{2}{|c|}{$1 A^{1}$ vs rest } \\
\hline ARF & 48 & $0(0.00)$ & $11(0.23)$ & $37(0.77)$ & 0.009 & 0.0017 & 0.004 & 0.005 \\
\hline No ARF & 22 & $2(0.09)$ & $10(0.46)$ & $10(0.46)$ & $0.25(0.08-0.73)$ & $0.08(0.02-0.39)$ & $0.23(0.11-0.68)$ & $0.21(0.07-0.62)$ \\
\hline ARDS & 16 & $0(0.00)$ & $2(0.12)$ & $14(0.88)$ & - & 0.040 & - & 0.021 \\
\hline No ARDS & 54 & $2(0.04)$ & $19(0.35)$ & $33(0.61)$ & - & $0.16(0.03-0.92)$ & - & $0.12(0.02-0.73)$ \\
\hline MV & 24 & $0(0.00)$ & $4(0.17)$ & $20(0.83)$ & 0.037 & 0.019 & 0.034 & 0.015 \\
\hline No MV & 46 & $2(0.04)$ & $17(0.37)$ & $27(0.59)$ & $0.28(0.08-0.97)$ & $0.08(0.01-0.66)$ & $0.31(0.1-0.96)$ & $0.16(0.04-0.70)$ \\
\hline$I C U^{e}$ & 30 & $0(0.00)$ & $4(0.13)$ & $26(0.87)$ & 0.003 & 0.015 & 0.003 & 0.029 \\
\hline No ICU & 40 & $2(0.05)$ & $17(0.43)$ & $21(0.53)$ & $0.17(0.05-0.58)$ & $0.25(0.08-0.76)$ & $0.20(0.07-0.62)$ & $0.32(0.11-0.89)$ \\
\hline
\end{tabular}

Only those comparisons with $P<0.05$ and significant odds ratio were included. OR $(95 \% \mathrm{Cl})$, odds ratio ( $95 \%$ confidence interval); ARF, acute respiratory failure; ARDS, acute respiratory distress syndrome; MV, mechanical ventilation; ICU, intensive care unit.

${ }^{a} P$ value for the bivariate comparison calculated with the $x^{2}$ test. ${ }^{b}+-+$ value for the multivariate analysis calculated with binary logistic regression, including the variables age, gender, risk factors, secondary bacterial pneumonia, and bacteremia. ${ }^{c} P$ value by Fisher Exact test. ${ }^{d} P$ value for the multivariate analysis calculated with binary logistic regression, including the variables age, gender, risk factors, and secondary bacterial pneumonia (bacteremia variable was excluded because it shows a co-lineal relation with the variable mechanical ventilation). ${ }^{~}$ Patients who required (ICU) or did not require (No ICU) ICU admission, $P$ value for the multivariate analysis calculated with conditional logistic regression stratified by hospital of origin, including the covariables age, gender, risk factors, secondary bacterial pneumonia, and bacteremia. Conventional haplotypes at SFTPA2 were identified on the basis of combinations of the polymorphism rs1059046 (T9N), rs17886395 (A91P), and rs1965708 (Q223K). "Rest" refers to the other haplotypes for each comparison.

the effect of the SFTPA2 variants on the need for ICU admission was detected in the first few days of hospitalization, as would be expected for components of the innate immunity involved in inflammation and defense against IAV infection.

The significance of the functional differences between variants at SFTPA1 and SFTPA2 is poorly understood $[5,15]$. The variant rs1965708 produces an amino-acid change at residue 223 (Q223K), located in the CRD of SP-A2, and might directly influence the binding properties to either IAV or the antiinflammatory receptor SIRPa. Residue 9 (rs1059046, T9N) is located in the signal peptide, and it is unknown whether this variant may affect the protein. It is, however, worth noting that haplotypes $1 A^{0}$ and $1 A^{1}$ differ precisely at residues 9 and 223. A role of SNPs at regulatory regions in haplotypes $1 A^{O}$ and $1 A^{1}$ in translation and/or RNA stability of SFTPA2 cannot be ruled out $[5,15]$. Interestingly, SP-A2 $1 \mathrm{~A}^{1}$ variants were shown to have a lower activity for enhancement of TNF- $\alpha$ in macrophage-derived THP-1 cells than other variants, such as $1 \mathrm{~A}$ and $1 \mathrm{~A}^{0}$, particularly after oxidative stress [28,29]. These data suggest that the protective effect of the SP-A2 $1 \mathrm{~A}^{1}$ variant in the severity of H1N1pdm infection could be due to its lower proinflammatory activity.

Irrespective of the causal $\mathrm{SNP}(\mathrm{s})$, our data suggest that the haplotype $1 A^{O}$ of SFTPA2 is associated with a higher severity after $\mathrm{H} 1 \mathrm{~N} 1 \mathrm{pdm}$ infection, whereas the SFTPA2 haplotype $1 A^{1}$ was associated with a dominant protective effect against severe forms of H1N1pdm infection.

We acknowledge several limitations of our study. First, the overrepresentation of hospitalized patients, could bias us to analyze susceptibility to H1N1pdm infection. In addition, our control group could be considered a representative sample from the Spanish population rather than a representation of non-H1N1pdm-infected individuals.

Second, our study is underpowered to detect the role of some variants on severity of H1N1pdm infection. Nevertheless, considering odds ratios and a significance level of 5\%, the power of the associations of rs1965708$C$ with the development of ARDS and septic shock was 


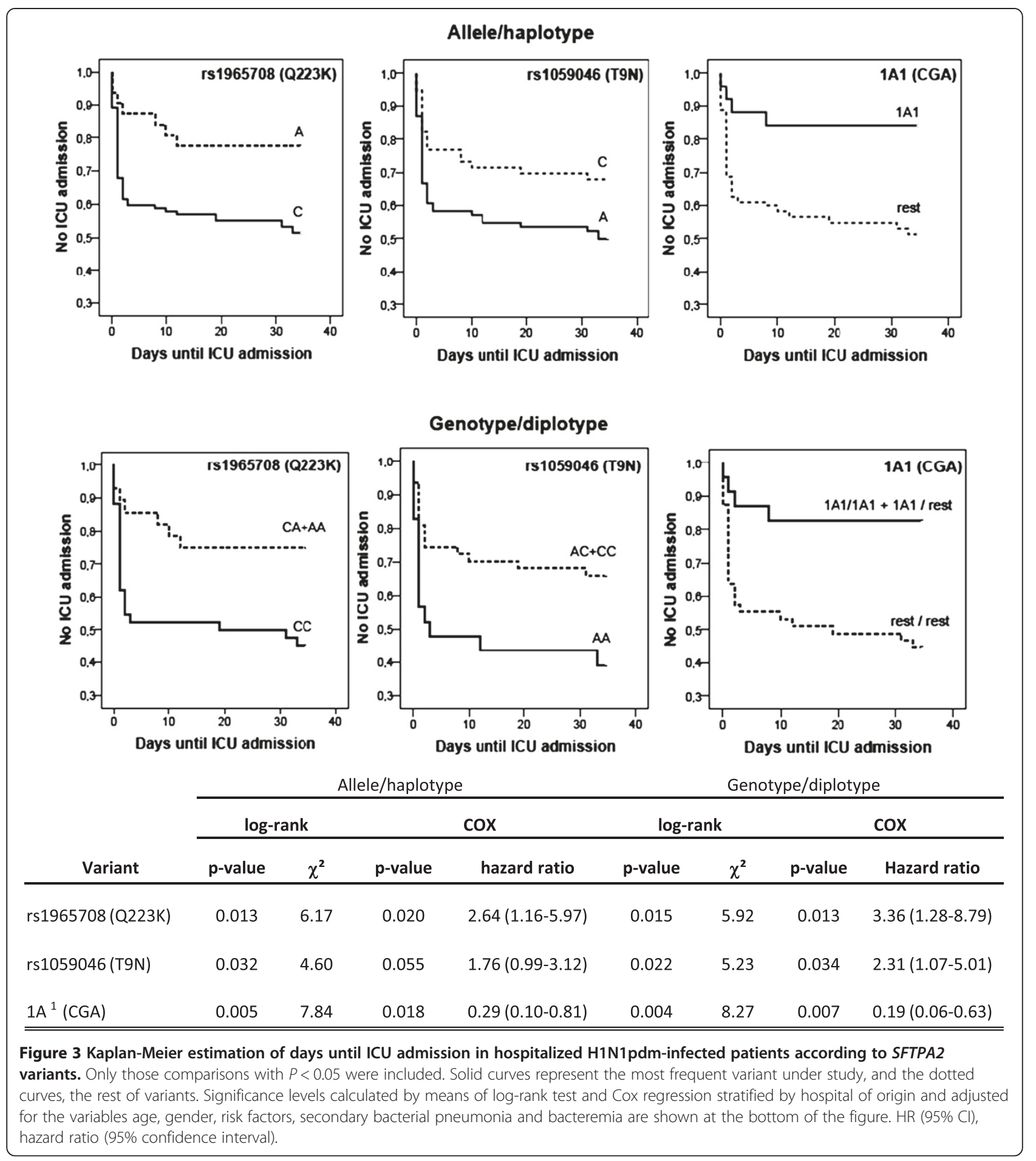

$84 \%$, and more than $88 \%$ for the need for MV. In the haplotype analysis, statistical power was $88.56 \%$ for the effect of the haplotype $1 A^{1}$ on ICU admission (90.62\% for the effect in a dominant model). No correction for multiple comparisons was performed in these comparisons, but, as expected by in vivo and in vitro studies among the human collectins, only SP-A would be expected to influence H1N1pdm infectivity, and significant associations were repeatedly observed with several clinical phenotypes. Furthermore, the observed associations were found to be independent of age, gender, risk factors predisposing to severe H1N1 infection, and development 

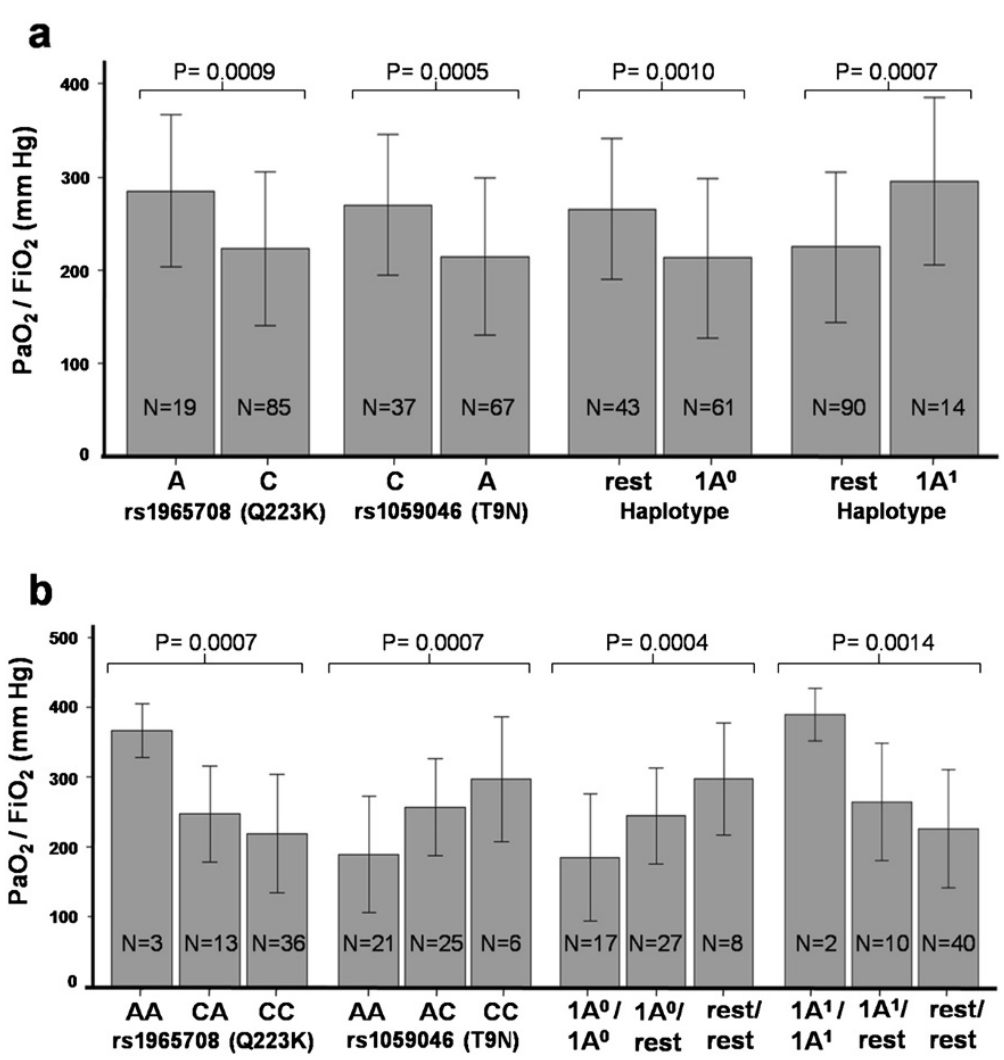

Figure 4 Ratio of oxygen arterial pressure to oxygen inspiratory fraction $\left(\mathrm{PaO}_{2} / \mathrm{FiO}_{2}\right)$ with regard to SFTPA2 genetic variants. PaO $/ \mathrm{FiO}_{2}$ with regard to alleles and haplotypes (a) as well as genotypes and diplotypes (b) of SFTPA2 in hospitalized patients with H1N1 pandemic 2009 influenza A virus infection. Each bar represents the mean \pm SD. P values were calculated with a regression lineal model, including the variables age, gender, risk factors, secondary bacterial pneumonia, and bacteremia.

of either secondary bacterial pneumonia or bacteremia. Variants of SFTPA2 were clearly associated with functional parameters of gas exchange, underscoring their role in the severity of H1N1pdm-induced lung injury.

Third, criteria for ICU admission may vary between different centers. To avoid these differences, multivariate and Cox regression analysis to evaluate the need for ICU admission were stratified by hospital of origin.

\section{Conclusion}

Our study suggests that variants at SFTPA2 influence the severity of H1N1pdm infection in hospitalized patients. The potential of collectins as therapeutic agents for the treatment of IAV-mediated disease is now being explored [7,30]. In Sftpa-/-and Sftpd-/-mice, intratracheally administered SP-A or SP-D can restore microbial clearance and inflammation [5], and exogenous surfactant preparations containing the hydrophobic SP-B and $-C$ are widely used for replacement therapies in pRDS. The data from our study, together with a better knowledge of the functional significance of the genetic variability at SFTPA2 on IAV-associated disease, could pave the way for a potential treatment with haplotypespecific $\left(1 A^{1}\right)$ SP-A2 for patients with the most severe forms of the disease in future IAV pandemics.

\section{Key messages}

- Genetic variation in the SFTPA2 gene influences the outcome of patients infected with the 2009 pandemic H1N1 influenza A virus.

- Data from our study may help to identify patients at higher risk of severe pandemic (nonglycosylated) IAV infection, who may eventually benefit from more-personalized and targeted therapies.

\section{Abbreviations}

ARDS: Acute respiratory distress syndrome; ARF: acute respiratory failure; BMI: body mass index; CEIC: Comité Ético de Investigación Clínica (Clinical Research Ethics Committee); CRD: carbohydrate recognition domain; $\mathrm{FiO}_{2}$ : fraction of inspired oxygen; $\mathrm{H} 1 \mathrm{~N} 1 \mathrm{pdm}$ : virus influenza $\mathrm{A} \mathrm{H} 1 \mathrm{~N} 1$ pandemic; HIV: human immunodeficiency virus; HR: hazard ratio; IAV: influenza A virus; ICU: intensive care unit; MBL: mannose-binding lectin; MODS: multiorgan dysfunction syndrome; MV: mechanical ventilation; NFKB: nuclear factor kappa $\mathrm{B}$; OR: odds ratio; $\mathrm{PaO}_{2}$ : partial pressure of oxygen; 
pRDS: respiratory distress syndrome in prematurely born infant; PVP: primary viral pneumonia; $\mathrm{SaO}_{2}$ : arterial oxygen saturation; SIRPa: signal inhibitory regulatory protein a; SNP: single-nucleotide polymorphism; SP: surfactant protein.

\section{Competing interests}

The author(s) declare that they have no competing interests.

\section{Authors' contributions}

EHR: acquisition, analysis, and interpretation of molecular genetic data, and statistical analysis and collaboration in the writing of the manuscript. MLR: acquisition, analysis, and interpretation of molecular genetic data and critical review of the manuscript. JJRH: acquisition, analysis, and interpretation of clinical data and critical review of the manuscript. JPH: acquisition, analysis, and interpretation of clinical data and critical review of the manuscript. LB: acquisition, analysis, and interpretation of clinical data and critical review of the manuscript. EL: acquisition, analysis, and interpretation of clinical data and critical review of the manuscript. JB: acquisition, analysis, and interpretation of clinical data and critical review of the manuscript. MCPG: acquisition, analysis, and interpretation of H1N1pdm infection data and critical review of the manuscript. MIGL: molecular genetic data acquisition, analysis, and interpretation and critical review of the manuscript. YF: molecular genetic data acquisition and critical review of the manuscript. VMB: acquisition, analysis, and interpretation of H1N1pdm infection data and critical review of the manuscript. MM: acquisition, analysis, and interpretation of clinical data and critical review of the manuscript. JMF: acquisition, analysis, and interpretation of clinical data and critical review of the manuscript. LS: acquisition, analysis, and interpretation of clinical data and critical review of the manuscript. CV: acquisition, analysis, and interpretation of H1N1pdm infection data and critical review of the manuscript. OR: acquisition, analysis, and interpretation of clinical data and critical review of the manuscript. MB: acquisition, analysis, and interpretation of clinical data and critical review of the manuscript. JA: acquisition, analysis, and interpretation of clinical data and critical review of the manuscript. ELG: acquisition, analysis, and interpretation of clinical data and critical review of the manuscript. JSV: acquisition, analysis, and interpretation of clinical data and collaboration in the writing of the manuscript. FRC: financial support, acquisition, analysis, and interpretation of clinical data and collaboration in the writing of the manuscript. CRG: coordination, conception and design, financial support, and manuscript writing. All authors read and approved the final version of the manuscript.

\section{Authors' information}

Jordi Solé-Violán and Felipe Rodríguez de Castro should be regarded as cosecond last senior authors.

\section{Acknowledgements}

We are grateful to the patients and their families for their trust, as well as to the healthy volunteers. We also thank Miguel Ángel García-Bello for statistical assistance, Nereida González-Quevedo for technical features, and Consuelo Ivañez for their invaluable help. We appreciate the attention given by local Ethics Committee of involved hospitals: CEIC Hospital Universitario de Gran Canaria Dr. Negrín, CEIC Hospital del Mar de Investigaciones Médicas, CEIC Hospital San Jorge, Hospital Clínico y CEIC Universitario de Valencia, and CEIC Hospital de la Princesa.

This work was supported by grants from Fondo de Investigaciones Sanitarias, Ministerio de Economía y Competitividad [PI 02/1620, 04/1190, 06/1031, 10/ 01718, and 12/01565] with the funding of European Regional Development Fund-European Social Fund (FEDER-FSE); RedRespira-ISCIII-RTIC-03/11; Sociedad Española de Neumología y Cirugía Torácica (SEPAR); E.H.R. and Y.F. by a grant from Universidad de Las Palmas de Gran Canaria, and M.L.R., by a grant from Instituto de Salud Carlos III, Ministerio de Economía y Competitividad [FI 11/00593]. The sponsors of the study had no role in designing the study, collecting, analyzing, and interpreting the data, or writing the manuscript.

\section{Author details}

${ }^{1}$ Department of Immunology, Hospital Universitario de Gran Canaria Dr. Negrín, Las Palmas de Gran Canaria 35010, Spain. ${ }^{2}$ Department of Medical and Surgical Sciences, School of Medicine, Universidad de Las Palmas de Gran Canaria, Las Palmas de Gran Canaria 35016, Spain. ${ }^{3}$ Department of Internal Medicine, Hospital Universitario de Gran Canaria Dr Negrín, Las
Palmas de Gran Canaria 35010, Spain. ${ }^{4}$ Department of Infectious Diseases, Hospital Universitari del Mar, Barcelona 08003, Spain. ${ }^{5}$ Hospital del Mar de Investigaciones Médicas (IMIM), CIBERES, Barcelona 08003, Spain.

${ }^{6}$ Department of Respiratory Diseases, Hospital San Jorge, Huesca 22004, Spain. ${ }^{7}$ Intensive Care Unit, Hospital Clínico y Universitario de Valencia, Valencia 46010, Spain. ${ }^{8}$ Department of Microbiology, Hospital Universitario de Gran Canaria Dr. Negrín, Las Palmas de Gran Canaria 35010, Spain. ${ }^{9}$ Center for Experimental and Molecular Medicine, Academic Medical Center, Amsterdam $1105 \mathrm{AZ}$, The Netherlands. ${ }^{10}$ Laboratori de Referència de Catalunya, Prat de Llobregat, Barcelona 08820, Spain. ${ }^{11}$ Intensive Care Unit, Hospital Universitario de Gran Canaria Dr. Negrín, CIBERES, Las Palmas de Gran Canaria 35010, Spain. ${ }^{12}$ Department of Respiratory Diseases, Hospital Universitario de la Princesa, Madrid 28005, Spain. ${ }^{13}$ Department of Respiratory Diseases, Hospital Clínico y Universitario de Valencia, Valencia 46010, Spain. ${ }^{14}$ Department of Immunology, Hospital La Paz, Madrid 28046, Spain. ${ }^{15}$ Department of Respiratory Diseases, Hospital Universitario de Gran Canaria Dr. Negrín, Las Palmas de Gran Canaria 35010, Spain.

Received: 8 November 2013 Accepted: 4 June 2014

Published: 20 June 2014

\section{References}

1. Bautista E, Chotpitayasunondh T, Gao Z, Harper SA, Shaw M, Uyeki TM, Zaki SR, Hayden FG, Hui DS, Kettner JD, Kumar A, Lim M, Shindo N, Penn C, Nicholson KG: Clinical aspects of pandemic 2009 influenza A (H1N1) virus infection. N Engl J Med 2010, 362:1708-1719.

2. Horby $P$, Nguyen NY, Dunstan SJ, Baillie JK: The role of host genetics in susceptibility to influenza: a systematic review. PLoS One 2012, 7:e33180.

3. Albright FS, Orlando P, Pavia AT, Jackson GG, Cannon Albright LA: Evidence for a heritable predisposition to death due to influenza. J Infect Dis 2008, 197:18-24.

4. Keynan Y, Malik S, Fowke KR: The role of polymorphisms in host immune genes in determining the severity of respiratory illness caused by pandemic H1N1 influenza. Public Health Genomics 2013, 16:9-16.

5. Wright JR: Immunoregulatory functions of surfactant proteins. Nat Rev Immunol 2005, 5:58-68.

6. Eisen DP: Mannose-binding lectin deficiency and respiratory tract infection. J Innate Immun 2010, 2:114-122.

7. Ng WC, Tate MD, Brooks AG, Reading PC: Soluble host defense lectins in innate immunity to influenza virus. J Biomed Biotechnol 2012, 2012:732191.

8. Selman L, Hansen S: Structure and function of collectin liver 1 (CL-L1) and collectin 11 (CL-11, CL-K1). Immunobiology 2012, 217:851-863.

9. Hawgood S, Brown C, Edmondson J, Stumbaugh A, Allen L, Goerke J, Clark $\mathrm{H}$, Poulain F: Pulmonary collectins modulate strain-specific influenza a virus infection and host responses. J Virol 2004, 78:8565-8572.

10. LeVine AM, Hartshorn K, Elliott J, Whitsett J, Korfhagen T: Absence of SP-A modulates innate and adaptive defense responses to pulmonary influenza infection. Am J Physiol Lung Cell Mol Physiol 2002, 282:L563-L572.

11. Li G, Siddiqui J, Hendry M, Akiyama J, Edmondson J, Brown C, Allen L, Levitt $S$, Poulain F, Hawgood S: Surfactant protein-A-deficient mice display an exaggerated early inflammatory response to a beta-resistant strain of influenza A virus. Am J Respir Cell Mol Biol 2002, 26:277-282.

12. Hartshorn KL, White MR, Shepherd V, Reid K, Jensenius JC, Crouch EC: Mechanisms of anti-influenza activity of surfactant proteins $A$ and $D$ : comparison with serum collectins. Am J Physiol 1997, 273:L1156-L1166.

13. Job ER, Deng YM, Tate MD, Bottazzi B, Crouch EC, Dean MM, Mantovani A, Brooks AG, Reading PC: Pandemic H1N1 influenza A viruses are resistant to the antiviral activities of innate immune proteins of the collectin and pentraxin superfamilies. J Immunol 2010, 185:4284-4291.

14. Mikerov AN, White $M$, Hartshorn $K$, Wang $G$, Floros J: Inhibition of hemagglutination activity of influenza A viruses by SP-A1 and SP-A2 variants expressed in CHO cells. Med Microbiol Immunol 2008, 197:9-12.

15. Silveyra P, Floros J: Genetic variant associations of human SP-A and SP-D with acute and chronic lung injury. Front Biosci 2012, 17:407-429.

16. Haczku A: Protective role of the lung collectins surfactant protein $A$ and surfactant protein D in airway inflammation. J Allergy Clin Immunol 2008, 122:861-879.

17. Hoover RR, Floros J: Organization of the human SP-A and SP-D loci at 10q22-q23: physical and radiation hybrid mapping reveal gene order and orientation. Am J Respir Cell Mol Biol 1998, 18:353-362. 
18. Garcia-Laorden MI, de Rodriguez CF, Sole-Violan J, Rajas O, Blanquer J, Borderias L, Aspa J, Briones ML, Saavedra P, Marcos-Ramos JA, GonzálezQuevedo N, Sologuren I, Herrera-Ramos E, Ferrer JM, Rello J, Rodríguez-Gallego C: Influence of genetic variability at the surfactant proteins $A$ and $D$ in community-acquired pneumonia: a prospective, observational, genetic study. Crit Care 2011, 15:R57.

19. Garcia-Laorden MI, de Rodriguez CF, Sole-Violan J, Payeras A, Briones ML, Borderias L, Aspa J, Blanquer J, Rajas O, Marcos-Ramos JA, Herrera-Ramos E, García-Bello MA, Noda J, Ferrer JM, Rello J, Rodríguez-Gallego C: The role of mannose-binding lectin in pneumococcal infection. Eur Respir J 2013, 41:131-139.

20. Bone RC, Balk RA, Cerra FB, Dellinger RP, Fein AM, Knaus WA, Schein RM, Sibbald WJ: Definitions for sepsis and organ failure and guidelines for the use of innovative therapies in sepsis: The ACCP/SCCM Consensus Conference Committee, American College of Chest Physicians/Society of Critical Care Medicine. Chest 1992, 101:1644-1655.

21. Bernard GR, Artigas A, Brigham KL, Carlet J, Falke K, Hudson L, Lamy M, Legall JR, Morris A, Spragg R, The American-European Consensus Conference on ARDS: Definitions, mechanisms, relevant outcomes, and clinical trial coordination. Am J Respir Crit Care Med 1994, 149:818-824.

22. DiAngelo S, Lin Z, Wang G, Phillips S, Ramet M, Luo J, Floros J: Novel, non-radioactive, simple and multiplex PCR-CRFLP methods for genotyping human SP-A and SP-D marker alleles. Dis Markers 1999, 15:269-281.

23. The International HapMap Consortium: A haplotype map of the human genome. Nature 2005, 437:1299-1320

24. Barrett JC, Fry B, Maller J, Daly MJ: Haploview: analysis and visualization of LD and haplotype maps. Bioinformatics 2005, 21:263-265.

25. Foreman MG, Kong X, DeMeo DL, Pillai SG, Hersh CP, Bakke P, Gulsvik A, Lomas DA, Litonjua AA, Shapiro SD, Tal-Singer R, Silverman EK: Polymorphisms in surfactant protein-D are associated with chronic obstructive pulmonary disease. Am J Respir Cell Mol Biol 2011, 44:316-322.

26. Lingappa JR, Dumitrescu L, Zimmer SM, Lynfield R, McNicholl JM, Messonnier NE, Whitney CG, Crawford DC: Identifying host genetic risk factors in the context of public health surveillance for invasive pneumococcal disease. PLoS One 2011, 6:e23413.

27. Job ER, Deng YM, Barfod KK, Tate MD, Caldwell N, Reddiex S, Maurer-Stroh S, Brooks AG, Reading PC: Addition of glycosylation to influenza A virus hemagglutinin modulates antibody-mediated recognition of H1N1 2009 pandemic viruses. J Immunol 2013, 190:2169-2177.

28. Wang G, Umstead TM, Phelps DS, Al-Mondhiry H, Floros J: The effect of ozone exposure on the ability of human surfactant protein a variants to stimulate cytokine production. Environ Health Perspect 2002, 110:79-84.

29. Mikerov AN, Umstead TM, Gan X, Huang W, Guo X, Wang G, Phelps DS, Floros J: Impact of ozone exposure on the phagocytic activity of human surfactant protein A (SP-A) and SP-A variants. Am J Physiol Lung Cell Mol Physiol 2008, 294:L121-L130.

30. Sato A, Whitsett JA, Scheule RK, Ikegami M: Surfactant protein-d inhibits lung inflammation caused by ventilation in premature newborn lambs. Am J Respir Crit Care Med 2010, 181:1098-1105.

doi:10.1186/cc13934

Cite this article as: Herrera-Ramos et al: Surfactant protein A genetic variants associate with severe respiratory insufficiency in pandemic influenza A virus infection. Critical Care 2014 18:R127.

\section{Submit your next manuscript to BioMed Central and take full advantage of:}

- Convenient online submission

- Thorough peer review

- No space constraints or color figure charges

- Immediate publication on acceptance

- Inclusion in PubMed, CAS, Scopus and Google Scholar

- Research which is freely available for redistribution

Submit your manuscript at www.biomedcentral.com/submit
Ciomed Central 